\title{
Adaptation of Tendon Structure and Function in Tendinopathy With Exercise and Its Relationship to Clinical Outcome
}

\author{
Kenneth Färnqvist, Stephen Pearson, and Peter Malliaras
}

\begin{abstract}
Context: Exercise is seen as the most evidence-based treatment for managing tendinopathy and although the type of exercise used to manage tendinopathy may induce adaptation in healthy tendons, it is not clear whether these adaptations occur in tendinopathy and if so whether they are associated with improved clinical outcomes. Objective: The aim of the study was to synthesize available evidence for adaptation of the Achilles tendon to eccentric exercise and the relationship between adaptation (change in tendon thickness) and clinical outcomes among people with Achilles tendinopathy. Evidence Acquisition: The search was performed in September 2018 in several databases. Studies investigating the response (clinical outcome and imaging on ultrasound/magnetic resonance imaging) of pathological tendons (tendinopathy, tendinosis, and partial rupture) to at least 12 weeks of eccentric exercise were included. Multiple studies that investigated the same interventions and outcome were pooled and presented in effect size estimates, mean difference, and 95\% confidence intervals if measurement scales were the same, or standard mean difference and 95\% confidence intervals if measurements scales were different. Where data could not be pooled the studies were qualitatively synthesized based on van Tulder et al. Evidence Synthesis: Eight studies met the inclusion and exclusion criteria and were included in the review. There was strong evidence that Achilles tendon thickness does not decrease in parallel with improved clinical outcomes. Conclusions: Whether a longer time to follow-up is more important than the intervention (ie, just the time per se) for a change in tendon thickness remains unknown. Future studies should investigate whether exercise (or other treatments) can be tailored to optimize tendon adaptation and function, and whether this relates to clinical outcomes.
\end{abstract}

Keywords: tendon adaptation, tendinitis, tendinosis, physical activity

Tendinopathy is a general term indicating any abnormal state of a tendon, including pain, dysfunction, and, but not necessarily, pahology. ${ }^{1,2}$ Pathological tendons are defined as having abnormal changes on imaging (eg, increased signal intensity on magnetic resonance imaging [MRI] or hypoechoic regions in ultrasound) or on histological investigations. ${ }^{2}$ Histological changes that can be seen in tendinopathy include increased water content, increased amounts of proteoglycans and glycosaminoglycans, fascicular disorganization, neurovascular ingrowth, and changes in cell phenotype. ${ }^{3,4}$ Pathological tendons (ie, those with imaging or histological abnormalities) may not necessarily be painful. Pain-free tendons with pathology are common and measures of structural changes, at any given time, cannot be used to determine whether a tendon is perceived painful. ${ }^{5}$

Tendon, like muscle, is a plastic tissue that adapts to ensure it can "cope" with habitual loading. This plasticity may be via transduction mechanisms, that is, the fibroblasts linking to the extracellular matrix via integrins, which enable the cell to detect and adapt to the mechanical loading. Tendon material properties or tendon structure (eg, increased fibril size, additional enzymatically derived cross-links between fibrils) and tendon morphology or size (eg, increased cross-sectional area [CSA], thickness) may adapt to

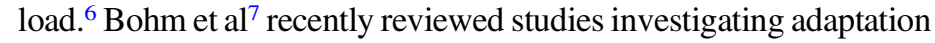
of healthy human tendon to mechanical loading and found consistent evidence for increased stiffness following high load interventions

Färnqvist is with Haninge Rehab, Handen, Sweden. Pearson is with the Centre for Health, Sport \& Rehabilitation Sciences Research, University of Salford, Manchester, United Kingdom. Malliaras is with the Department of Physiotherapy, School of Primary Health Care, Faculty of Medicine, Nursing and Health Science, Monash University, Melbourne, VIC, Australia. Färnqvist (k.farnqvist@gmail.com) is corresponding author. that bring about large tendon strains ( $>70 \%$ maximal voluntary contraction or $>70 \% 1$ repetition maximum).

The question that now arises is whether a change in tendon structure (ie, tendon size) occurs in tendinopathy following exercise interventions and whether these changes are associated with clinical symptoms. Exercise is seen as the most common evidence-based treatment for managing tendinopathy, ${ }^{8}$ and although the type of exercise used to manage tendinopathy may induce adaptation in healthy tendons, it is not clear whether these adaptations occur in tendinopathy and if so whether they are associated with improved clinical outcomes. A prior systematic review ${ }^{9}$ did not support a relationship between observable structural change (MRI and ultrasound) and improved clinical outcomes when treated by eccentric exercise, but this was based on imaging (structural changes, eg, echogenicity) outcomes with poor reliability, ${ }^{10}$ bringing into question the validity of the conclusions. A further systematic review ${ }^{11}$ concluded that there is moderate evidence for an association between clinical outcomes and tendon thickness and tendon neovascularization in tendinopathy, but included cointerventions with exercise that may influence tendon adaptation (eg, injection therapy). Given the uncertainty in the literature and methodological limitations, a review that focuses on reliable imaging outcomes and exercise interventions without confounding cointerventions is warranted. This will allow athletic trainers and other clinicians to make informed decisions about the potential mechanisms to target when delivering exercise interventions for people with Achilles tendinopathy.

\section{Objective}

The aim of this review is to synthesize current evidence that reports a change in tendon structure (change in tendon thickness) in 
Achilles tendinopathy in response to exercise ( $>12 \mathrm{wk})$ and whether this relates to change in clinical outcomes (self-reported pain and function). The focus is on reliable outcomes of a change in tendon structure and studies measuring eccentric exercise from single-cohort studies as well as those studies comparing eccentric exercise with another intervention or wait and see. This review may inform clinical practice and future studies regarding optimal exercise interventions to achieve a change in tendon structure.

\section{Evidence Acquisition}

Inclusion Criteria. Randomized controlled trials (RCTs), controlled trials, cohort studies, and case series were included. Studies investigating reliable measures of a change in tendon structure (outcome) following at least 12 weeks of exercise (interventions) among participants with Achilles tendinopathy were included. An additional outcome measure criterion was that studies needed to include at least 1 validated outcome measure for self-reported pain and/or function (eg, Victorian Institute of Sport Assessment-Achilles Questionnaire, Numeric Rating Scale).

Study Type. Randomized controlled trial design (eg, parallel group, factorial, cross-over), pseudo-randomized trials, and singlecohort studies were included. There were no restrictions based on date of publication.

Population. We included studies investigating participants with Achilles tendinopathy of any age, and duration of symptoms of at least 3 months. Only studies that clearly defined clinical diagnostic criteria for tendon-related pain (localized [insertion or midportion], Achilles tendon pain for $>3$ months, corresponding tenderness on palpation, thickening of the Achilles tendon 2 to $6 \mathrm{~cm}$ above the tendon insertion [for midportion only]), and demonstrated tendon pathology on ultrasound (tendon thickening, hypoechoic regions, and/or Doppler signal), MRI (tendon thickening and increased signal), or ultrasound tissue characterization (increase in abnormal echo types) were included. Studies also needed to exclude other potential causes of pain and interventions that could moderate tendon structure change following exercise such as systemic disease (eg, rheumatoid arthritis, psoriatic arthritis, or inflammatory bowel disease), positive neurological tests, reproduction of pain from movement of proximal joints (eg, back movement reproducing leg or foot pain), steroid injection in the last 3 months, and previous surgery to the affected tendon or joint.

Tendon Structure Outcomes. Includes tendon thickness or CSA measured with ultrasound or MRI. Measures of tendon thickness and CSA have demonstrated acceptable reliability. ${ }^{12-14}$
Interventions. Studies including exercise as a treatment or rehabilitation for Achilles tendinopathy were included. The only additional parameter was that exercise needed to be applied for 12 weeks or longer as a shorter intervention does not seem to bring about structural changes in the (healthy) tendon. ${ }^{7}$ Any exercise frequency, load intensity, time under tension, and rest times were included with the exception of contraction type where only studies investigating eccentric exercise were included. Studies investigating other types of exercise (eg, heavy slow resistance [HSR]) were excluded because of the low number of studies and thus the ability to systematically review the literature.

Exclusion Criteria. Reviews, case studies, and opinion articles were excluded, as were animal and non-English language studies. Studies investigating tendon rupture/full thickness tears (eg, postsurgical intervention exercise) as well as those investigating other tendons that were not the Achilles tendon were excluded because their number was too low. Studies investigating nonexercise interventions alone (eg, manual therapy, taping, shockwave) were also excluded. Studies investigating shockwave or injections were excluded because they may influence a change in tendon structure. ${ }^{15-17}$ Stretching was not considered an exercise intervention that is able to lead to a change in chronic tendon structure $^{7}$ and studies that investigated only stretching were thus excluded. Studies with cointerventions were excluded, as although they were unlikely to influence a change in tendon structure (eg, manual therapy or tape), they could influence the experience of pain. We also excluded studies investigating biomarkers because although exercise increases tendon collagen synthesis, ${ }^{18}$ this biochemical response may not necessarily translate to tendon material or structural properties. ${ }^{19}$ Studies investigating other types of tendon adaptation (eg, tendon mechanical properties, echo types investigated with ultrasound tissue characterization) were excluded too because of the low number of studies and thus the ability to systematically review the literature.

Search Strategy. The preferred reporting items for systematic reviews and meta-analyses statement protocol guidelines were followed in this systematic review. ${ }^{20}$ The search was performed in November 2018 in the following databases: MEDLINE, CINAHL, EBSCO, and Google Scholar. Only terms relating to the intervention and population were searched. Adding the outcome resulted in a very low yield because most studies did not mention a change in tendon structure in the title, abstract, or keywords. No limits were applied (see Table 1 for MEDLINE example and subject headings for CINAHL and EBSCO). Several studies in the field of tendinopathy and the reference lists of final yield were screened to identify other eligible studies.

Review Process. Two reviewers (KF and PM) screened all titles and after excluding studies both abstracts and titles were screened and further studies were excluded. The full text of the remaining

\section{Table 1 Search Terms in Database}

\begin{tabular}{ll}
\hline Search term & Synonyms \\
\hline Exercise (intervention) & $\begin{array}{l}\text { Concentric, eccentric, isometric, heavy slow, load, physiotherapy, physical therapy, and strength training } \\
\text { (title and abstract); exercise, exercise therapy, rehabilitation, resistance training (MeSH), concentric contraction, } \\
\text { eccentric contraction, isometric contraction, exercise, physical therapy, resistance training, and rehabilitation (subject } \\
\text { heading CINAHL); and exercise, exercise therapy, rehabilitation, and resistance training (subject heading EBSCO) }\end{array}$ \\
$\begin{array}{l}\text { Achilles, tendo-Achilles, tricep-surae, triceps, and triceps tendon (title and abstract); Achilles tendon (MeSH) } \\
\text { (subject heading CINAHL); and Achilles tendon (subject heading EBSCO) }\end{array}$ \\
$\begin{array}{l}\text { AND patholion) } \\
\text { (population) }\end{array}$ & $\begin{array}{l}\text { Paratendinitis, pathological, and partial rupture (title and abstract); pathology, tendinopathy (MeSH) pathology, } \\
\text { tendinopathy, and Achilles tendinopathy (subject heading CINAHL); and tendinopathy (subject heading EBSCO) }\end{array}$ \\
\hline
\end{tabular}


studies was screened. A third reviewer (SP) was also available to discuss eligibility of studies when this was not clear.

Risk of Bias Assessment. The risk of bias was evaluated by 2 independent assessors (KF and $\mathrm{PM}$ ) and disagreements were resolved via consensus and adjudicated by a third author (SP) where necessary. Given that an RCT is the most appropriate design to answer our question, study quality was assessed using the Physiotherapy Evidence Database (PEDro) scale, ${ }^{21}$ which has established reliability and validity. ${ }^{21,22}$ The advantage of using the PEDro scale is that it penalizes cohort design studies where confounders such as regression to mean and natural recovery may influence outcomes. ${ }^{21}$ The scale has 11 criteria (1 point per criterion) with a maximum score of 10 because the first question is not marked. A study with a low risk of bias was defined as having $\geq 6 / 10$ score. $^{21}$

Data Extraction. Data were extracted independently by 2 reviewers ( $\mathrm{KF}$ and $\mathrm{PM}$ ), using a recommended form. ${ }^{20} \mathrm{~A}$ third reviewer (SP) was available to adjudicate when there was lack of consensus. Data extracted included study design, participant characteristics (including midportion or insertional tendinopathy), pathological tendon decrease, intervention, tendon structure outcome, and clinical outcome (eg, Victorian Institute of Sport AssessmentAchilles Questionnaire, Numeric Rating Scale). If there was more than 1 measurement scale used for the same construct, we included scales that were similar across most studies; otherwise we prioritized validated and disease-specific questionnaires. Outcome data were extracted for dichotomous (proportions and sample size) and continuous data (follow-up or change scores, SD, and sample size) to enable calculation of effect sizes (see "Data Synthesis and Analysis" section below). We extracted data at the follow-up times where tendon structure and clinical outcomes were both reported.

Data Synthesis and Analysis. We planned to perform metaanalyses among trials with similar characteristics (exercise interventions and comparator, outcome measures, and populations). ${ }^{23}$ We planned to use random effects models, as clinical and methodological heterogeneity is likely to exist and may impact model findings. Where data could not be pooled, we qualitatively synthesized findings based on van Tulder et $\mathrm{al}^{24}$ levels of evidence (Table 2). Although the synthesis according to van Tulder et $\mathrm{al}^{24}$ should be based on RCTs and clinical controlled trials, it has often been used for other study designs. , $^{95,26}$ Positive change in tendon thickness was defined as change toward the comparator group. Pathological Achilles tendons are reported to be thicker, ${ }^{27}$ so a positive change is expected to be reduced thickness.

Where there were multiple studies (trials and single-cohort studies) that investigated the same interventions and outcome we calculated effect size estimates. Dichotomous outcomes were expressed as relative risk and $95 \%$ confidence intervals. Continuous

\section{Table 2 Level of Evidence ${ }^{24}$}

\begin{tabular}{ll}
\hline $\begin{array}{l}\text { Level of } \\
\text { evidence }\end{array}$ & Measures \\
\hline Strong & $\begin{array}{l}\text { Consistent findings in multiple high-quality studies } \\
(\mathrm{n}>2)\end{array}$ \\
Moderate & $\begin{array}{l}\text { Consistent findings among multiple low-quality } \\
\text { studies or 1 high-quality study }\end{array}$ \\
Limited & $\begin{array}{l}\text { Findings from 1 low-quality study. } \\
\text { Conflicting }\end{array}$ \\
No evidence & \begin{tabular}{l} 
No studies found \\
\hline
\end{tabular}
\end{tabular}

outcomes were expressed as mean difference (MD) and 95\% confidence intervals if measurement scales were the same, or as standard mean difference and $95 \%$ confidence intervals if measurements scales were different. Standard mean difference effect sizes were interpreted as small (0.2), medium (0.5), and large $(0.8){ }^{28}$ The standard mean difference was adjusted (Hedges' adjusted $g$ ) for small sample bias. ${ }^{23}$

\section{Evidence Synthesis}

Search Yield. The search results are shown in Figure 1. Eight studies met the inclusion and exclusion criteria and were included in the review. Two studies ${ }^{29,30}$ used the same cohort and investigated different follow-up times. Data from these studies were combined, so overall 8 studies and 7 unique cohorts were included.

Risk of Bias. Four studies had low risk of bias ${ }^{31-34}$ and 4 had high risk of bias ${ }^{29,30,35,36}$ (see Table 3). The mean risk of bias score was just over 5 out of 10 (5.3) and scores ranged between 2 and 8 . The most common quality criteria not satisfied was blinding of subjects and therapists.

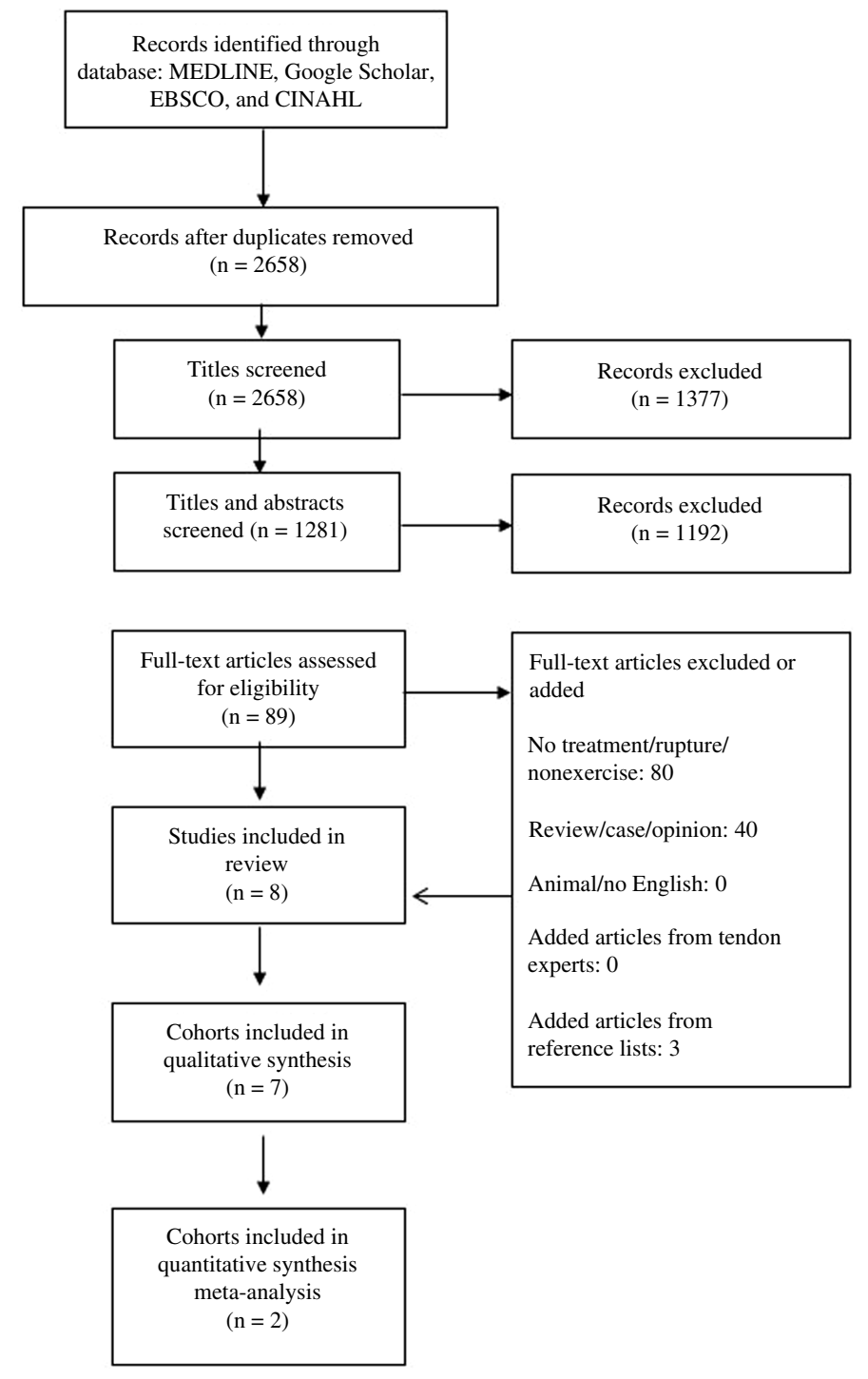

Figure 1 - Study selection flow chart. 


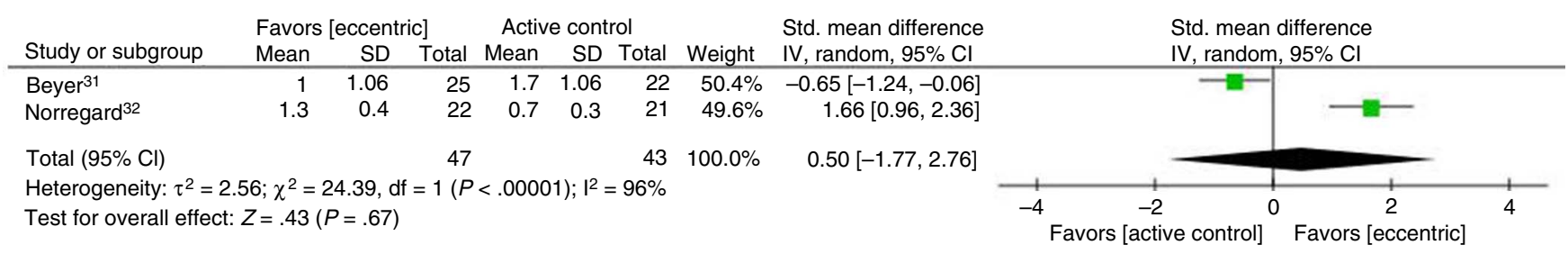

Figure 2 - Comparison of thickness outcomes measured by ultrasound between eccentric exercise (Alfredson protocol) and a heavy slow exercise protocol or stretching. CI, confidence interval; Std., standard.

Table 3 PEDro Scale-Risk of Bias Assessment of Included Studies

\begin{tabular}{|c|c|c|c|c|c|c|c|c|c|c|c|c|}
\hline Study & EC & RTG & $A C$ & SB & BS & BT & BA & KO & ITT & BG & MV & TS \\
\hline Beyer $^{31}$ & Yes & Yes & Yes & Yes & No & No & Yes & No & Yes & Yes & Yes & 7 \\
\hline Gärdin'29 & Yes & No & No & No & No & No & Yes & Yes & Yes & No & Yes & 4 \\
\hline Norregard ${ }^{32}$ & Yes & Yes & Yes & No & No & No & Yes & No & Yes & Yes & Yes & 6 \\
\hline Petersen $^{33}$ & Yes & Yes & Yes & Yes & No & No & Yes & No & Yes & Yes & Yes & 7 \\
\hline Richards $^{35}$ & Yes & N/A & N/A & N/A & N/A & No & Yes & No & Yes & No & No & 2 \\
\hline Rompe $^{34}$ & Yes & Yes & Yes & Yes & No & No & Yes & Yes & Yes & Yes & Yes & 8 \\
\hline Shalabi ${ }^{30}$ & Yes & No & No & No & No & No & Yes & No & Yes & No & Yes & 3 \\
\hline Tsahie $^{36}$ & Yes & No & No & Yes & No & No & No & Yes & Yes & Yes & Yes & 5 \\
\hline
\end{tabular}

Abbreviations: AC, allocation was concealed; BA, blinding of all assessors; BG, between-group statistical; BS, blinding of all subjects; BT, blinding of all therapists; EC, eligibility criteria; ITT, intention to treat; KO, key outcome was obtained; MV, measures of variability; RTG, randomly allocated to groups; SB, similar at baseline; TS, Total score.

Study Characteristics. Characteristics of included studies are shown in Table 4. Most studies were RCTs ${ }^{31-34}$ or prospective cohort studies. ${ }^{29,30,36}$ There was 1 controlled clinical trial. ${ }^{35}$ There were a total of 321 participants (173 men and 148 women) with mean age of 48 years (ranging from $42^{32}$ to 51 ), ${ }^{30}$ and mean duration of symptoms of 14 months.

Eight studies ( 7 cohorts) investigated eccentric training. ${ }^{29-36}$ Eccentric training involves 3 sets of 15 isolated eccentric exercises, both with straight and bent knee, performed twice daily. ${ }^{31}$ Pain and discomfort are acceptable, and additional weight is added when the exercise becomes pain free. All studies executed the Alfredson protocol with exceptions from 2 studies. ${ }^{33,36}$ In the study by Petersen et $\mathrm{al}^{33}$ the participants did the exercises 3 times per day and in the study by Tsehaie et $\mathrm{al}^{36}$ the participants had a familiarization period for 4 weeks before the actual exercise period for 12 weeks started.

Only 2 studies ${ }^{31,32}$ qualified for meta-analyses comparing eccentric exercise versus heavy slow exercise ${ }^{31}$ or stretching ${ }^{32}$ and the analyses showed an $\mathrm{I}^{2}$ of $96 \%$, so we decided to present the individual results from each study (Table 5) as well as pooled data (Figure 2).

Tendon Size Changes and Clinical Outcomes Findings with Eccentric Exercise in Achilles Tendinopathy. Thickness, pain, and function outcomes: There is strong evidence to refute that Achilles tendon thickness decreases (normalization) in parallel with improvement in self-reported pain and function. Four cohorts with low risk of bias (all RCTs) ${ }^{31-34}$ found either no change in parallel with improved self-reported clinical outcomes. There were heterogeneous between group differences with benefit of eccentric training compared to stretching (large effect) and inferior change in thickness of eccentric training compared to heavy slow resistance (moderate effect). Three cohorts with high risk of bias (all cohort studies) found either no change in tendon thickness ${ }^{29,30,36}$ in parallel with improved self-reported clinical outcomes or that symptoms improved before the Achilles size reduced. ${ }^{35}$ Effect sizes were calculated and pooled where possible (see Table 5, Figure 2).

\section{Discussion}

The aim of this review was to synthesize current evidence that reports a change in tendon structure (change in tendon thickness) in Achilles tendinopathy in response to exercise $(>12 \mathrm{wk})$ and whether this relates to change in clinical outcomes (self-reported pain and function). We found that there was strong evidence that Achilles tendon thickness does not decrease in parallel with improved clinical outcomes. After 12 weeks of eccentric exercise only 2 studies $^{31,32}$ showed effect sizes not crossing zero regarding tendon thickness, but these showed heterogeneous differences between groups (compared with HSR $^{31}$ and stretching ${ }^{32}$ ). Further, the study by Norregard et $\mathrm{al}^{32}$ was the only study that included participants with insertional tendinopathy, which could have affected the outcomes. The included cohort studies either reported no change in thickness at follow-up ${ }^{29,30,36}$ or found that symptoms improved before the Achilles size reduced. ${ }^{35}$

Our findings suggest that the inconsistent changes seen in tendon thickness could be explained by time rather than eccentric exercise. However, in our effect size calculations we found that eccentric exercise was more effective $[1.66(0.96,2.36)]$ than stretching in the study by Norregard, ${ }^{32}$ but the study by Beyer et $\mathrm{al}^{31}$ found no difference between both exercise groups (eccentric vs HSR) which suggests that loading maybe of importance. However, even in the original study by Norregard et al, ${ }^{32}$ there was no significant difference between groups, but no exact $P$ values or effect sizes were presented. The review by Rabello ${ }^{11}$ concluded that there is moderate evidence for an association between clinical outcomes and tendon thickness and tendon neovascularization in 


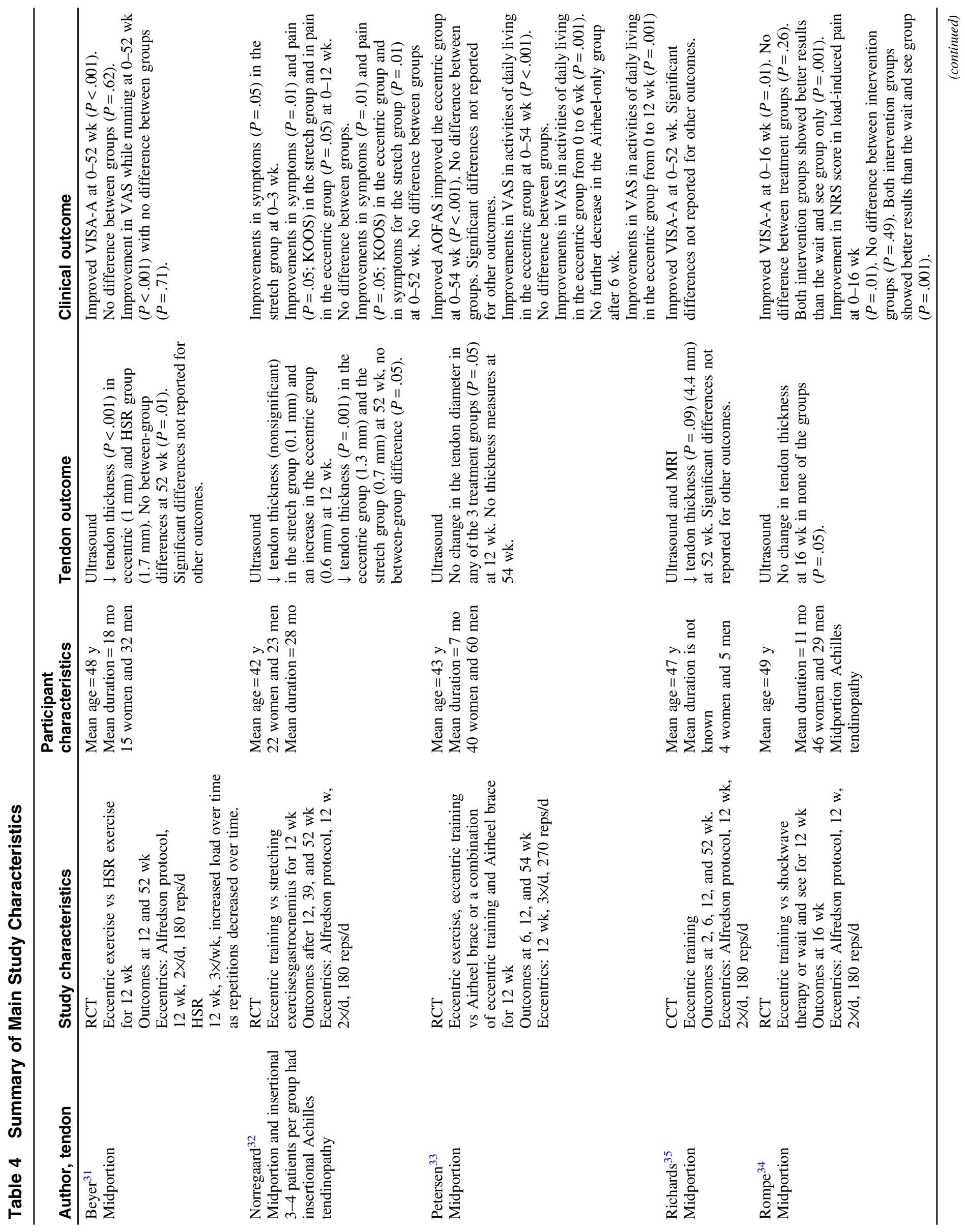




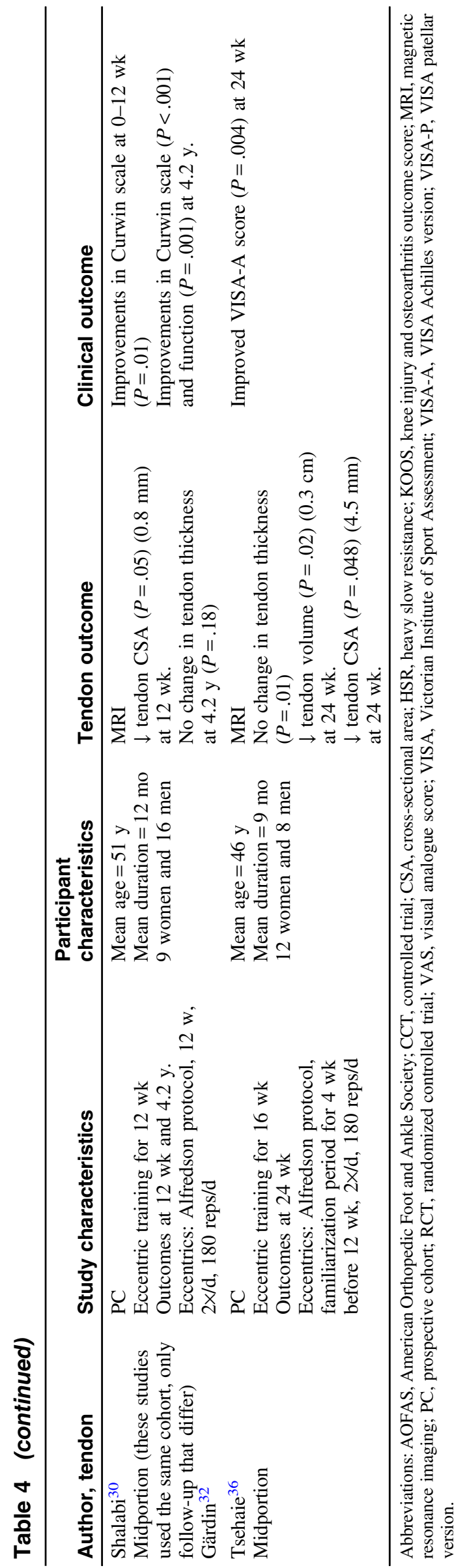

tendinopathy, which is the opposite of what we conclude. The difference between this study and the 2 earlier reviews ${ }^{9,11}$ is the methodology and we only investigated eccentric exercise (alone without any cointervention) by itself or compared with another intervention where it became clear that the number of studies, and therefore, the overall evidence, is scarce.

\section{Comparison With Earlier Evidence}

In the Drew et $\mathrm{al}^{9}$ systematic review, it was concluded that HSR exercise may be superior to other exercises in relation to tendon adaptation outcomes. This was based on a report of superior Doppler signal and tendon thickness outcomes with HSR compared with eccentric exercise in the Kongsgaard et $\mathrm{al}^{37}$ patellar tendinopathy study. In Achilles tendinopathy, which was the focus of this review, a recent study compared HSR versus eccentric exercise and found no difference in tendon thickness change or improvements in clinical outcomes between the groups. ${ }^{31}$ Based on these limited data, there is a lack of evidence (Achilles) that HSR is superior at adapting pathological tendon, but more evidence is needed before firm conclusions can be made. Theoretically, HSR may involve heavier load than the Alfredson eccentric program (up to 6 repetition maximum compared with up to 15 repetition maximum in eccentric exercise). However, there is no way of knowing whether this is the case because adherence and fidelity of either program are not reported, and the HSR program was performed double leg and the eccentric program single leg.

\section{Change in Tendon Structure With Exercise and Relationship to Clinical Outcome}

There was within group clinical benefit in pain and function with exercise which was not paralleled with change in tendon thickness. Only one study found a between-group difference. Rompe et $\mathrm{al}^{34}$ found that eccentric exercise or shockwave therapy improved Victorian Institute of Sport Assessment-Achilles version and NRS scores more than the wait and see group. However, no exercise regime seems to be superior regarding clinical outcomes in both Achilles and patellar tendinopathy patients. ${ }^{38}$ To the authors' knowledge, there is only one study investigating fibril morphology and clinical outcomes. This study ${ }^{39}$ found that fibril morphology became more normal (increase fibril density and decrease mean area) following HSR exercise in patellar tendinopathy. Although this suggests a relationship between microstructural tendon adaptation and clinical outcomes, caution is needed as the data are from only one study. Given that this is the only study to investigate tendon microstructure with biopsy, it raises the possibility that current imaging measures lack sufficient sensitivity to identify tendon adaptations that may be occurring in tendinopathy. In support of this, in the same study, the authors reported no change in tendon CSA, modulus, or stiffness. ${ }^{39}$ A potential limitation of the biopsy procedure is that it could have contributed to changes in the tendon (eg, collagen type III and type I collagen expression). ${ }^{40}$

\section{Tendon Thickness}

Unlike healthy tendon where change in thickness or CSA may indicate increased collagen content, change in pathological tendon thickness is more likely to indicate change in bound water and proteoglycan content. ${ }^{41}$ Tendon thickening and increased proteoglycan content are an attempt to reduce permeability and fluid flow and load on tendon cells. ${ }^{42}$ Preliminary evidence from the studies 
Table 5 Effect Size Pre-Post Eccentric Intervention on Tendon Thickness

\begin{tabular}{lccccc}
\hline Study & $\begin{array}{c}\text { PEDro } \\
\text { score }\end{array}$ & $\begin{array}{c}\text { Length of } \\
\text { follow-up }\end{array}$ & $\begin{array}{c}\text { Thickness in mm (SD) } \\
\text { at baseline-follow-up }\end{array}$ & $\begin{array}{c}\text { Thickness in mm (SD) } \\
\text { at follow-up }\end{array}$ & $\begin{array}{c}\text { Between-group } \\
\text { difference (SMD) }\end{array}$ \\
\hline Rompe $^{34}$ & 8 & $16 \mathrm{wk}$ & $12.8(4.1)$ & None & None \\
Tsahie $^{36}$ & 5 & $24 \mathrm{wk}$ & $8.9-8.6$ & Not reported & No comparison group \\
Beyer $^{31}$ & 7 & $52 \mathrm{wk}$ & $8.3(1.06)$ & $7.3(1.06)$ & $-0.65(-1.24,-0.06)$ \\
Norregard $^{32}$ & 6 & $52 \mathrm{wk}$ & $7.6($ not reported) & $10.6(4.63)$ & No comparison group \\
Richards $^{35}$ & 2 & $52 \mathrm{wk}$ & $15(5.15)$ & None & None \\
Petersen $^{33}$ & 7 & $54 \mathrm{wk}$ & $6.4(2.0)$ & No comparison group \\
Shalabi/Gärdin & 29,30 & 4 & $4.2 \mathrm{y}$ & $6.7(2.0)$ & $6.96,2.36)$ \\
\hline
\end{tabular}

Abbreviation: SMD, standard mean difference.

included in this review suggests that the Achilles tendon does not change in thickness in parallel with improved clinical outcome in Achilles tendinopathy. Whether change in thickness is a mechanism for change in pain and the clinical relevance of a few millimeters' reduction in thickness are unknown. It is noteworthy that some tendons may increase collagen content and size, but reduce water content after an exercise intervention so overall their thickness does not change and in this case adaptation may only be detected by assessing mechanical properties. A recent study ${ }^{43}$ investigating the long-term prognosis (5-15 y follow-up) in plantar fascia-related pain found a greater decrease than studies included in this review (in asymptomatic and symptomatic groups from 6.9 and $6.7 \mathrm{~mm}$, respectively, to $4.3 \mathrm{~mm}$ in both groups). However, at follow-up, fascia thickness and echogenicity had normalized in only $24 \%$ of the patients in the asymptomatic group and fascia thickness decreased over time regardless of symptoms and had no impact on prognosis.

Tendon thickness has been shown to normalize after nonload interventions, ${ }^{44}$ so clearly other mechanisms may explain change in this outcome. Maybe a longer time to follow-up is more important than the intervention (ie, just the time per se)? Another example, the stage of pathology, may also influence change in tendon thickness but is not considered by any study in this review. ${ }^{45}$ For example, patellar tendons of volleyball players that were defined as "degenerative" based on the continuum model ${ }^{45}$ on gray-scale ultrasound imaging (hypoechoic regions and poor matrix alignment) were less likely to demonstrate change in tendon thickness over the course of a competitive season. ${ }^{46}$

\section{Incorporation of Evidence into Clinical Practice}

This review found that the clinical outcomes improved with time, but there is still a great uncertainty whether tendon thickness changes in parallel with change in symptoms. Recent evidence suggests that different types of pathology may be less likely to resolve over time ${ }^{46}$ and less responsive to exercise interventions, ${ }^{47}$ suggesting that mechanisms for change in patient symptoms following exercise most likely extend beyond a change in tendon structure. However, there is a possibility that current imaging methods are not sensitive enough to identify a change in tendon structure. What the limited evidence base suggests is that a change in tendon thickness following exercise seems different in pathological versus healthy tendons.

\section{Recommendations for Future Research}

Bohm et $\mathrm{al}^{7}$ demonstrated in their systematic review that thickness of healthy tendon consistently increases following HSR and heavy isometric interventions over a number of weeks, where the interventions with longer duration $(\geq 12 \mathrm{wk})$ seem to be more efficient to stimulate tendon change in several aspects. No study included in our review investigated eccentric exercise for more than 12 weeks and maybe this was the parameter missing for a change in tendon thickness. No studies utilizing elastography or more traditional measurement (ie, ultrasound) to investigate change in mechanical properties after exercise were identified. Given the lack of studies, no conclusions can be made about the relationship between change in mechanical properties with exercise and improvement in clinical outcomes in Achilles tendinopathy. Future studies should investigate whether pathological tendons respond to different exercise interventions (eg, applied for a longer period) or whether they have limited capacity for improvement in their mechanical properties. Finally, we acknowledge that stage of pathology as described in the continuum model ${ }^{45}$ may influence tendon adaptation ${ }^{46}$ and was not defined in studies in this review. However, it is important to note there is only prospective evidence for the continuum model utilizing imaging changes in patella tendons ${ }^{46}$ alongside a RCT where participants with Achilles tendinopathy were divided by pathology (reactive vs. degenerative), to examine the effects of supplements and exercise on pathology and patient outcomes. ${ }^{47}$ Whether change in tendon thickness is a mechanism explaining change in clinical outcome requires further investigation. Future studies should consider longer follow-up times and detailed reporting of exercise parameters (eg, load) to further elucidate the relationship between exercise adaptation (not only thickness) of pathological tendon mechanical properties in relation to clinical reporting outcomes. Considering other tendons (patellar, rotator cuff, and plantar fascia) and outcomes (tendon structure and mechanical properties), there is no clear evidence that a change in tendon structure improves in parallel with improved clinical outcomes due to lack of studies.

\section{Limitations}

The key disadvantage of this review is there were limited studies utilizing eccentric training in Achilles tendinopathy. Thus, the only tendon and contraction type investigated in this review were the Achilles tendon and eccentric exercise. There was also limited description of load intensity and compliance, so it is not possible to draw firm conclusions regarding the relationship between load and tendon structure of pathological tendon. It is very possible that many exercise protocols did not achieve an adequate intensity for a change in tendon structure. ${ }^{7}$ Further, the results from the single-arm studies should be interpreted with caution because the improvement cannot be differentiated from the confounders such as natural 
history, ${ }^{48}$ and this is reflected in our quality assessment. A further limitation is that the included studies report group averages, not individual data points. For example, individual data points are required to determine whether, in a given patient, tendon size and clinical outcome are trending in the same direction or not. Even if a study shows that, on group average, tendon thickness is decreasing and clinical outcomes are improving, the data do not demonstrate that these processes are occurring in the same individuals.

\section{Conclusions}

For Achilles tendinopathy, 12 weeks of eccentric exercise appears to result in improved clinical outcomes (self-reported pain and function) over time, but this often happened in the absence of reduced tendon thickness. This review has progressed current knowledge by identifying this lack of relationship between a change in tendon structure in pathological tendon and clinical outcomes, which is observed in many other areas of the body. Overall, we conclude that we cannot say with any certainty that pathological Achilles tendon thickness do change with load.

\section{Acknowledgment}

The authors declare no conflict of interest.

\section{References}

1. Rio E, Moseley L, Purdam C, et al. The pain of tendinopathy: physiological or pathophysiological? Sports Med. 2014;44(1): 9-23. PubMed ID: 24027089 doi:10.1007/s40279-013-0096-z

2. van Dijk CN, van Sterkenburg MN, Wiegerinck JI, Karlsson J, Maffulli N. Terminology for Achilles tendon related disorders. Knee Surg Sports Traumatol Arthrosc. 2011;19:835-841. PubMed ID: 21222102 doi:10.1007/s00167-010-1374-z

3. Dean BJ, Gettings P, Dakin SG, Carr AJ. Are inflammatory cells increased in painful human tendinopathy? A systematic review. $\mathrm{Br} J$ Sports Med. 2016;50:216-220. PubMed ID: 26246419 doi:10.1136/ bjsports-2015-094754

4. Riley G. Chronic tendon pathology: molecular basis and therapeutic implications. Expert Rev Mol Med. 2005;7:1-25. PubMed ID: 15796783 doi: $10.1017 /$ S1462399405008963

5. Giombini A, Dragoni S, Di Cesare A, Di Cesare M, Del Buono A, Maffulli N. Asymptomatic Achilles, patellar, and quadriceps tendinopathy: a longitudinal clinical and ultrasonographic study in elite fencers. Scand J Med Sci Sports. 2013;23:311-316. PubMed ID: 22092963 doi:10.1111/j.1600-0838.2011.01400.x

6. Miller BF, Olesen JL, Hansen M, et al. Coordinated collagen and muscle protein synthesis in human patella tendon and quadriceps muscle after exercise. J Physiol. 2005;567:1021-1033. PubMed ID: 16002437 doi:10.1113/jphysiol.2005.093690

7. Bohm S, Mersmann F, Arampatzis A. Human tendon adaptation in response to mechanical loading: a systematic review and metaanalysis of exercise intervention studies on healthy adults. Sports Med Open. 2015;1:7. PubMed ID: 27747846 doi:10.1186/s40798015-0009-9

8. Scott A, Docking S, Vicenzino B, et al. Sports and exercise-related tendinopathies: a review of selected topical issues by participants of the second International Scientific Tendinopathy Symposium (ISTS) Vancouver 2012. Br J Sports Med. 2013;47:536-544. PubMed ID: 23584762 doi:10.1136/bjsports-2013-092329
9. Drew BT, Smith TO, Littlewood C, Sturrock B. Do structural changes (eg, collagen/matrix) explain the response to therapeutic exercises in tendinopathy: a systematic review. Br J Sports Med. 2014;48:966972. PubMed ID: 23118117 doi:10.1136/bjsports-2012-091285

10. Collinger JL, Gagnon D, Jacobson J, Impink BG, Boninger ML. Reliability of quantitative ultrasound measures of the biceps and supraspinatus tendons. Acad Radiol. 2009;16(11):1424-1432. PubMed ID: 19596592 doi:10.1016/j.acra.2009.05.001

11. Rabello LM, van den Akker-Scheek I, Brink MS, Maas M, Diercks RL, Zwerver J. Association between clinical and imaging outcomes after therapeutic loading exercise in patients diagnosed with Achilles or patellar tendinopathy at short- and long-term follow-up: a systematic review [published online ahead of print June 25, 2018]. Clin J Sport Med.

12. Shalabi A, Movin T, Kristoffersen-Wiberg M, Aspelin P, Svensson L. Reliability in the assessment of tendon volume and intratendinous signal of the Achilles tendon on MRI: a methodological description. Knee Surg Sports Traumatol Arthrosc. 2005;13:492-498. PubMed ID: 16170584 doi:10.1007/s00167-004-0546-0

13. Silbernagel KG, Shelley K, Powell S, Varrecchia S. Extended field of view ultrasound imaging to evaluate Achilles tendon length and thickness: a reliability and validity study. Muscles Ligaments Tendons J. 2016;6:104-110. PubMed ID: 27331037 doi:10.32098/ mltj.01.2016.12

14. Mc Auliffe S, Mc Creesh K, Purtill H, O'sullivan K. A systematic review of the reliability of diagnostic ultrasound imaging in measuring tendon size: is the error clinically acceptable? Phys Ther Sport. 2017;26:52-63. PubMed ID: 28162938 doi:10.1016/j.ptsp.2016. 12.002

15. von Wehren L, Blanke F, Todorov A, Heisterbach P, Sailer J, Majewski M. The effect of subacromial injections of autologous conditioned plasma versus cortisone for the treatment of symptomatic partial rotator cuff tears. Knee Surg Sports Traumatol Arthrosc. 2016;24(12):3787-3792. PubMed ID: 26017742 doi:10.1007/ s00167-015-3651-3

16. Bosch G, Lin YL, van Schie HT, van De Lest CH, Barneveld A, van Weeren PR. Effect of extracorporeal shock wave therapy on the biochemical composition and metabolic activity of tenocytes in normal tendinous structures in ponies. Equine Vet J. 2007;39(3): 226-231. PubMed ID: 17520973 doi:10.2746/042516407X180408

17. Bosch G, de Mos M, van Binsbergen R, van Schie HT, van de Lest $\mathrm{CH}$, van Weeren PR. The effect of focused extracorporeal shock wave therapy on collagen matrix and gene expression in normal tendons and ligaments. Equine Vet J. 2009;41(4):335-341. PubMed ID: 19562893 doi: $10.2746 / 042516409 X 370766$

18. Langberg H, Ellingsgaard H, Madsen T, et al. Eccentric rehabilitation exercise increases peritendinous type I collagen synthesis in humans with Achilles tendinosis. Scand J Med Sci Sports. 2007;17:61-66. PubMed ID: 16787448

19. Magnusson SP, Langberg H, Kjaer M. The pathogenesis of tendinopathy: balancing the response to loading. Nat Rev Rheumatol. 2010;6: 262-268. PubMed ID: 20308995 doi:10.1038/nrrheum.2010.43

20. Liberati A, Altman DG, Tetzlaff J, et al. The PRISMA statement for reporting systematic reviews and meta-analyses of studies that evaluate healthcare interventions: explanation and elaboration. BMJ. 2009;339:b2700. PubMed ID: 19622552 doi:10.1136/bmj.b2700

21. de Morton NA. The PEDro scale is a valid measure of the methodological quality of clinical trials: a demographic study. Aust J Physiother. 2009;55:129-133. PubMed ID: 19463084 doi:10.1016/ S0004-9514(09)70043-1

22. Maher CG, Sherrington C, Herbert RD, Moseley AM, Elkins M. Reliability of the PEDro scale for rating quality of randomized 
controlled trials. Phys Ther. 2003;83(8):713-721. PubMed ID: 12882612

23. Deeks JJ, Higgins JPT, Altman DG, eds. Chapter 9: Analysing data and undertaking meta-analyses. In: Higgins JPT, Green S, eds. Cochrane Handbook for Systematic Reviews of Interventions Version 5.1.0 (updated March 2011). The Cochrane Collaboration; 2011. Retrieved from www.cochrane-handbook.org

24. van Tulder M, Furlan A, Bombardier C, Bouter L. Editorial board of the cochrane collaboration back review G. Updated method guidelines for systematic reviews in the Cochrane collaboration back review group. Spine. 2003;28:1290-1299.

25. Littlewood C, May S, Walters S. Epidemiology of rotator cuff tendinopathy: a systematic review. Shoulder \& Elbow. 2013;5(4): 256-265.

26. Mallows A, Debenham J, Walker T, Littlewood C. Association of psychological variables and outcome in tendinopathy: a systematic review. Br J Sports Med. 2017;51(9):743-748. PubMed ID: 27852585 doi:10.1136/bjsports-2016-096154

27. Docking SI, Ooi CC, Connell D. Tendinopathy: is imaging telling us the entire story? J Orthop Sports Phys Ther. 2015;45(11):842-852. PubMed ID: 26390270 doi:10.2519/jospt.2015.5880

28. Cohen J. Statistical Power Analysis for the Behavioral Sciences. 2nd ed. Hillsdale, NJ: Lawrence Erlbaum Associates; 1988.

29. Gardin A, Movin T, Svensson L, Shalabi A. The long-term clinical and MRI results following eccentric calf muscle training in chronic Achilles tendinosis. Skeletal Radiol. 2010;39:435-442. PubMed ID: 19774375 doi:10.1007/s00256-009-0798-3

30. Shalabi A, Kristoffersen-Wilberg M, Svensson L, Aspelin P, Movin T. Eccentric training of the gastrocnemius-soleus complex in chronic Achilles tendinopathy results in decreased tendon volume and intratendinous signal as evaluated by MRI. Am J Sports Med. 2004;32:1286-1296. PubMed ID: 15262655 doi:10.1177/ 0363546504263148

31. Beyer R, Kongsgaard M, Hougs Kjaer B, Ohlenschlaeger T, Kjaer M, Magnusson SP. Heavy slow resistance versus eccentric training as treatment for Achilles tendinopathy: a randomized controlled trial. Am J Sports Med. 2015;43:1704-1711. PubMed ID: 26018970 doi:10.1177/0363546515584760

32. Norregaard J, Larsen CC, Bieler T, Langberg H. Eccentric exercise in treatment of Achilles tendinopathy. Scand J Med Sci Sports. 2007;17:133-138. PubMed ID: 17394474

33. Petersen W, Welp R, Rosenbaum D. Chronic Achilles tendinopathy: a prospective randomized study comparing the therapeutic effect of eccentric training, the AirHeel brace, and a combination of both. Am J Sports Med. 2007;35:1659-1667. PubMed ID: 17569792 doi:10.1177/0363546507303558

34. Rompe JD, Nafe B, Furia JP, Maffulli N. Eccentric loading, shockwave treatment, or a wait-and-see policy for tendinopathy of the main body of tendo Achillis: a randomized controlled trial. Am J Sports Med. 2007;35:374-383. PubMed ID: 17244902 doi:10.1177/ 0363546506295940

35. Richards PJ, McCall IW, Day C, Belcher J, Maffulli N. Longitudinal microvascularity in Achilles tendinopathy (power Doppler ultrasound, magnetic resonance imaging time-intensity curves and the Victorian Institute of Sport Assessment-Achilles questionnaire): a pilot study. Skeletal Radiol. 2010;39(6):509-521. PubMed ID: 19711073 doi:10.1007/s00256-009-0772-0
36. Tsehaie J, Poot DHJ, Oei EHG, Verhaar JAN, de Vos RJ. Value of quantitative MRI parameters in predicting and evaluating clinical outcome in conservatively treated patients with chronic midportion Achilles tendinopathy: a prospective study. J Sci Med Sport. 2017; 20:633-637. doi:10.1016/j.jsams.2017.01.234

37. Kongsgaard M, Kovanen V, Aagaard P, et al. Corticosteroid injections, eccentric decline squat training and heavy slow resistance training in patellar tendinopathy. Scand J Med Sci Sports. 2009;19:790-802. PubMed ID: 19793213 doi:10.1111/j.1600-0838.2009.00949.x

38. Malliaras P, Barton CJ, Reeves ND, Langberg H. Achilles and patellar tendinopathy loading programmes: a systematic review comparing clinical outcomes and identifying potential mechanisms for effectiveness. Sports Med. 2013;43(4):267-286. PubMed ID: 23494258 doi:10.1007/s40279-013-0019-Z

39. Kongsgaard M, Qvortrup K, Larsen J, et al. Fibril morphology and tendon mechanical properties in patellar tendinopathy: effects of heavy slow resistance training. Am J Sports Med. 2010;38:749-756. PubMed ID: 20154324 doi:10.1177/0363546509350915

40. Heinemeier M, Lorentzen MP, Jensen J, et al. Local trauma in human patellar tendon leads to widespread changes in the tendon gene expression. J Appl Physiol. 2016;120:1000-1010. PubMed ID: 26769953 doi:10.1152/japplphysiol.00870.2015

41. Corps AN, Robinson AH, Movin T, Costa ML, Hazleman BL, Riley GP. Increased expression of aggrecan and biglycan mRNA in Achilles tendinopathy. Rheumatology. 2006;45:291-294. doi:10. 1093/rheumatology/kei152

42. Docking SI, Rosengarten SD, Cook J. Achilles tendon structure improves on UTC imaging over a 5-month pre-season in elite Australian football players. Scand J Med Sci Sports. 2016;26: 557-563. PubMed ID: 25943892 doi:10.1111/sms. 12469

43. Hansen L, Krogh TP, Ellingsen T, Bolvig L, Fredberg U. Long-term prognosis of plantar fasciitis: a 5- to 15-year follow-up study of 174 patients with ultrasound examination. Orthop J Sports Med. 2018;6(3):2325967118757983. PubMed ID: 29536022 doi:10. $1177 / 2325967118757983$

44. Horstmann T, Jud HM, Frohlich V, Mundermann A, Grau S. Wholebody vibration versus eccentric training or a wait-and-see approach for chronic Achilles tendinopathy: a randomized clinical trial. J Orthop Sports Phys Ther. 2013;43:794-803. PubMed ID: 24175595 doi:10.2519/jospt.2013.4762

45. Cook JL, Purdam CR. Is tendon pathology a continuum? A pathology model to explain the clinical presentation of load-induced tendinopathy. Br J Sports Med. 2009;43:409-416. PubMed ID: 18812414 doi:10.1136/bjsm.2008.051193

46. Malliaras P, Cook J. Changes in anteroposterior patellar tendon diameter support a continuum of pathological changes. Br J Sports Med. 2011;45:1048-1051. PubMed ID: 21540193 doi:10.1136/bjsm. 2010.082180

47. Balius R, Alvarez G, Baro F, et al. A 3-arm randomized trial for Achilles tendinopathy: eccentric training, eccentric training plus a dietary supplement containing mucopolysaccharides, or passive stretching plus a dietary supplement containing mucopolysaccharides. Curr Ther Res Clin Exp. 2016;78:1-7. PubMed ID: 28053674 doi:10.1016/j.curtheres.2016.11.001

48. Begg CB, Pilote L. A model for incorporating historical controls in into a meta-analysis. Biometrics. 1991;47(3):899-906. PubMed ID: 1742445 doi: $10.2307 / 2532647$ 